\title{
The role of laparoscopy in vascular surgery : our experience
}

\author{
K B Galketiya, V Pinto, R Perera, U Jayasooriya, R Rohankumar, D S B Gamage, \\ W N L Weerasinghe
}

\section{Teaching Hospital Peradeniya, Sri Lanka}

Abstract
The morbidity of open vascular surgery has been reduced by laparoscopic exposure. We present a case report of a patient who underwent laparoscopic assisted ilio-femoral bypass. The exposure of the aorto-iliac segment was performed laparoscopically. The anastomosis of the graft to the iliac artery was done via a mini incision. The bypass to the femoral artery was done using a standard groin incision.

Key words: Laparoscopy; vascular surgery

\section{Introduction}

Even though the history of vascular surgery originates from 1900, laparoscopy assisted vascular procedures were only reported in 1993. Since then this minimally invasive approach has gained popularity for both occlusive and aneurysmal conditions with the advantages of less postoperative pain, early ambulation and reduced hospital stay.

External iliac artery occlusion may be treated with angioplasty and stenting or by open bypass. Open bypass from the aorta or the common iliac artery to the femoral artery requires a substantial incision. Laparoscopic dissection allows exposure of the vessels with small port site incisions. The anastomosis may be done using intracorporeal suturing or a mini incision $(1,2,3)$.

We present a 44-year-old male who underwent a laparoscopy assisted ilio-femoral bypass.

\section{Case report}

A 44- year -old male smoker presented with rest pain and gangrene of the left 4 th and 5 th toes. He gave a history of intermittent claudication of ipsilateral thigh and calf. He had no history of ischaemic heart disease, cerebral ischaemia or mesenteric angina. On examination he had a weak left femoral pulse and an iliac bruit. His popliteal, posterior tibial and dorsalis pedis pulses were absent. His ankle-brachial pressure index (ABPI) was 0.3. Digital subtraction angiography (DSA) showed a narrowing of the left external iliac artery and occlusion of the left superficial femoral artery. There were multiple Correspondence : Galketiya KB

Tel :0777 884008

Email :kbgalketiya@yahoo.com

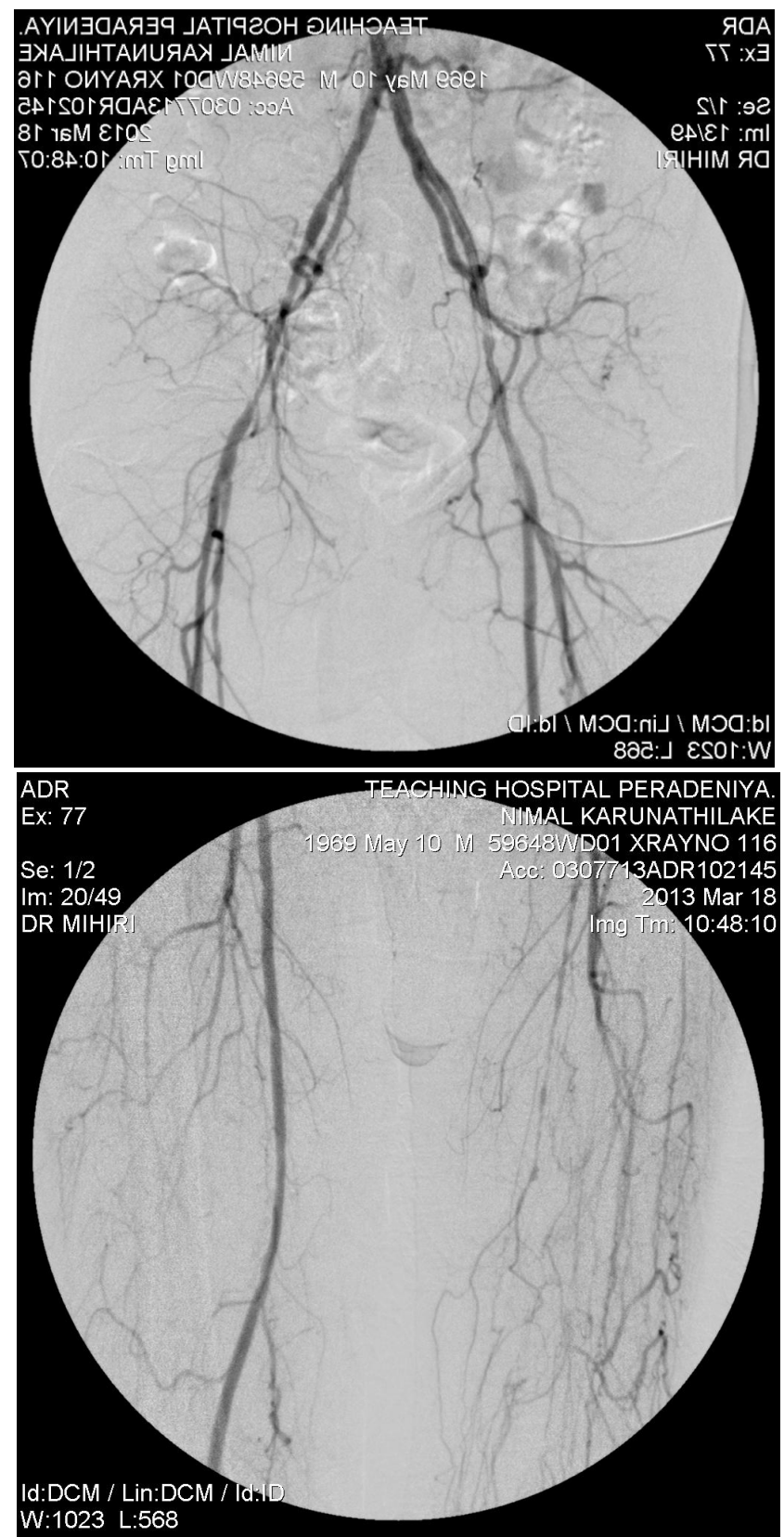




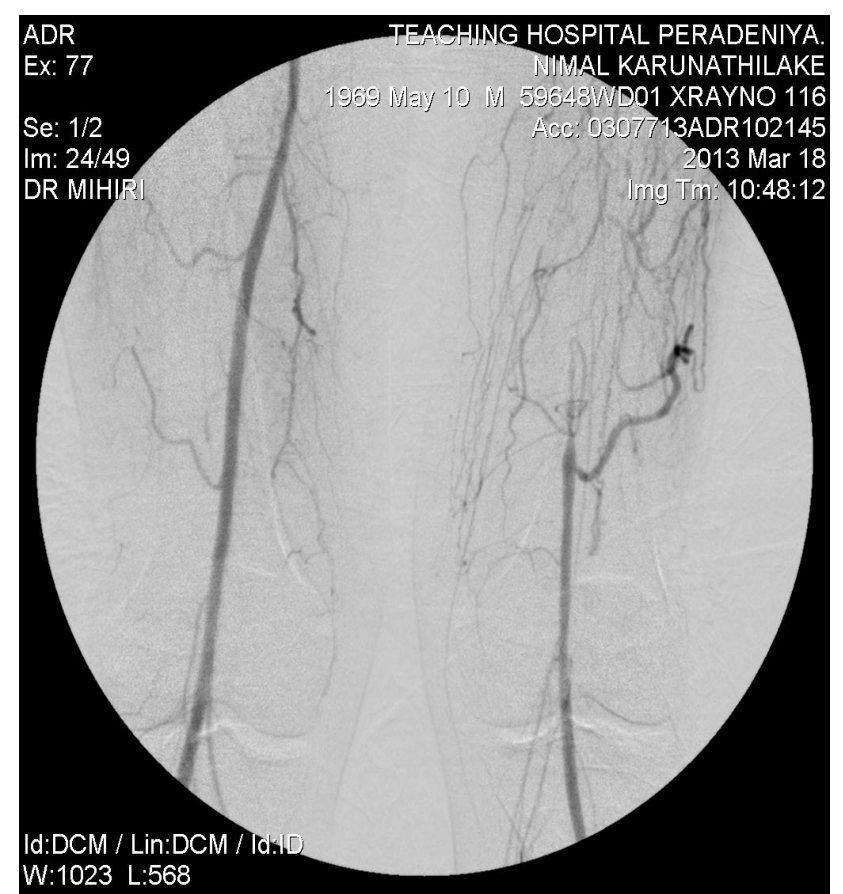

Figure 1-DSA images showing left external iliac artery narrowing and occlusion of the left superficial femoral artery.

The management of choice in this case was an angioplasty of the left iliac artery followed by a femoro-popliteal bypass. However, the short supply of accessories required for angioplasty necessitated bypass surgery. It was decided to perform an iliofemoral by pass and proceed to a femoro-popliteal bypass as a second stage, if necessary.

Surgery was performed under general anaesthesia. The patient was positioned in the right lateral and head low position. The surgical team operated from the right of the patient with the monitor on the left. Four ports were used. The sigmoid and descending colon were mobilized exposing the retroperitoneal tissues. A fan retractor introduced via a $5 \mathrm{~mm}$ port was used to keep the mobilized sigmoid colon in place. The bifurcation of the aorta, the common, external and internal iliac arteries were dissected. The left ureter was mobilized. Satisfactory pulsation of the common iliac artery was observed. Vascular clamps used in conventional open surgery were introduced via two stab incisions and the common iliac artery was clamped (Figure 2).

A $5 \mathrm{~cm}$ mini incision was made and the common iliac artery was opened longitudinally. An $8 \mathrm{~mm}$ synthetic graft was sutured end to side using 5-0 polypropylene. The entire procedure took two hours with no measurable blood loss.

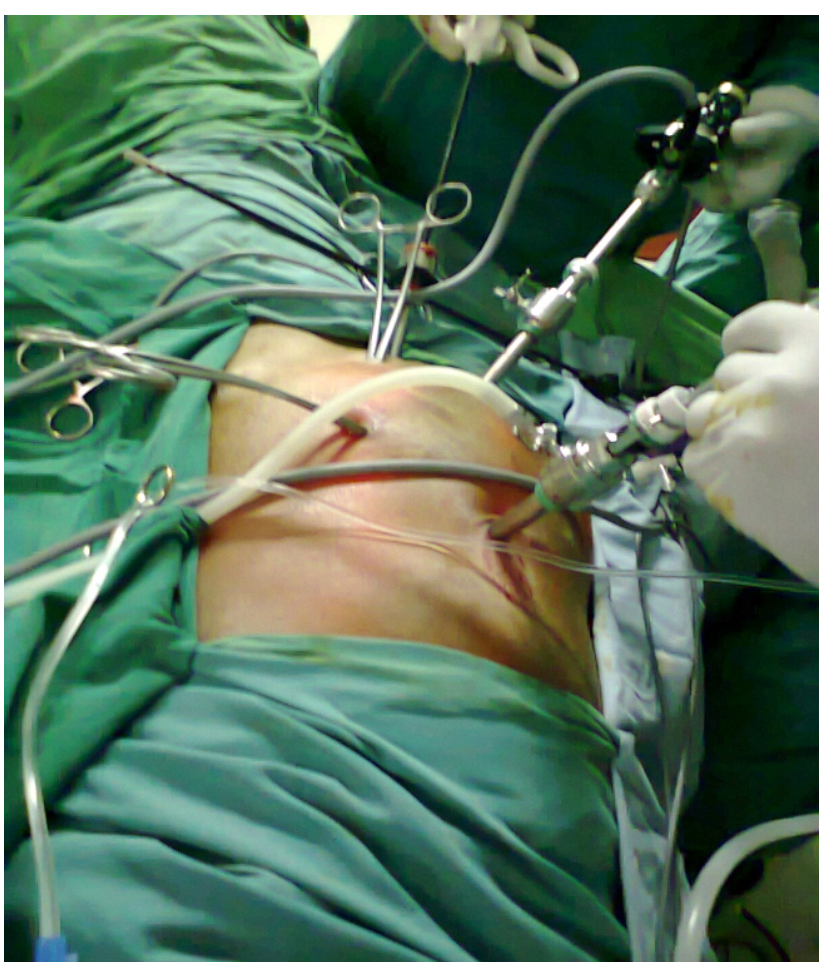

Figure 2. Ports and vascular clamps introduced via stab incisions.

The common femoral artery was exposed in the groin and the ilio-femoral bypass was completed. The immediate post-operative ABPI was 0.6. He was relieved of his rest pain. He required narcotic analgesics for postoperative pain for 24 hours and was subsequently managed with oral analgesics. Duplex assessment of the graft after one week showed a good flow.

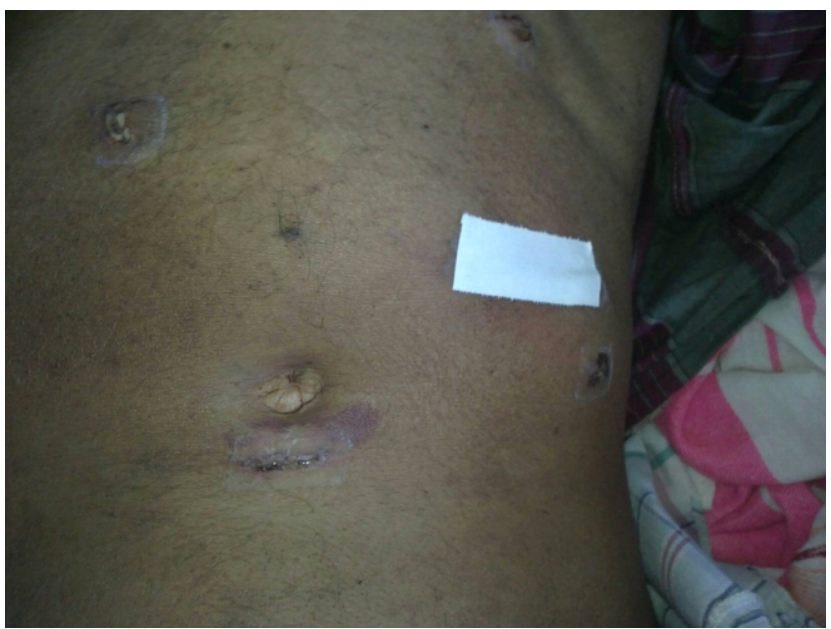

Figure 3. Postoperative scars 


\section{Discussion}

This is the first report of a laparoscopically assisted vascular bypass procedure reported in Sri Lanka. As demonstrated by others internationally and in this patient, in appropriately selected cases it reduces postoperative pain, enhances recovery and contributes to a shorter hospital stay.

\section{References}

1.Dion YM, Gracia CR, Estakhri M, Demalsy JC, Douville Y, Piccinini E, Stancanelli V Totally laparoscopic aortobifemoral bypass: a review of 10 patients. Surg Laparosc Endosc. 1998 Jun;8(3):16570 .

2.Cau J, Ricco JB, Corpataux JM.J Laparoscopic aortic surgery: Techniques and results Vasc Surg. 2008 Dec;48(6 Suppl):37S-44S

3.Berens ES, Herde JRJ, Laparoscopic vascular surgery: four case reports. Vasc Surg.; 1995 Jul;22(1):73-9. 